\title{
COPD: Reha nach Exazerbation sinnvoll
}

\author{
2011 prüfte ein Cochrane-Review die Evidenz für die \\ positiven Effekte einer pneumologischen Rehabili- \\ tation nach akuter COPD-Exazerbation. Ein großes \\ aktuelles Update bestätigt die Ergebnisse.
}

\section{Hier steht eine Anzeige.}

— Internationale Guidelines empfehlen für COPD-Patienten nach einer akuten Exazerbation (AE) eine pneumologische Rehabilitation. Das Cochrane-Institut hat nun sein systematisches Review aus dem Jahr 2011 aktualisiert. Gefunden wurden 11 neue randomisierte, kontrollierte Studien, sodass insgesamt 20 Studien mit 1.477 Probanden in die Analyse einflossen.

Im Hinblick auf das Risiko von Krankenhauseinweisungen nach einer AE schnitt die Reha signifikant besser ab als die Standardbehandlung (Odds Ratio 0,44, p=0,03). Beim Mortalitätsrisiko gab es keinen signifikanten Unterschied. Dafür verbesserte sich die Lebensqualität in der Reha-Gruppe im St. George's Respiratory Questionnaire hochsignifikant deutlicher, nämlich um 7,6 Punkte mehr ( $\mathrm{p}<0,001)$. Das gleiche galt für die körperliche Leistungsfähigkeit: Die Reha-Patienten schafften in der 6-Minuten-Gehteststrecke im Schnitt 62 Meter mehr $(\mathrm{p}<0,001)$.

Das Update bestätigt somit auf höchstem Evidenzniveau die Verbesserung von körperlicher Leistungsfähigkeit und Lebensqualität, und zwar nicht nur hochsignifikant, sondern auch klinisch relevant. Da einige neuere Studien aber keinen Benefit einer pneumologischen Reha auf erneute Krankenhauseinweisungen oder Mortalität zeigten, wurde die Evidenz für diese Parameter abgeschwächt.

- Puhan MA et al. Pulmonary rehabilitation following exacerbations of chronic obstructive pulmonary disease. Cochrane Database Syst Rev. 2016;12:CD005305

\section{KOMMENTAR}

Studien haben gezeigt, dass eine kurze 6-Minuten-Gehteststrecke bei COPD-Patienten mit einem deutlich erhöhten Mortalitätsrisiko verknüpft ist. Die minimal klinisch relevante Veränderung wird aktuell bereits ab 30 Metern angenommen, die Verbesserung von 62 Metern ist also klinisch relevant. Es darfangenommen werden, dass Patienten nach der Reha die Aktivitäten des täglichen Lebens wieder besser bewältigen und körperlich aktiver sein können.

Obwohl die Evidenz einer pneumologischen Rehabilitation nach einer AE bei COPD sehr gut ist, wird sie in Deutschland nur sehr selten verordnet. Nach Schätzungen wird eine solche Maßnahme nur 4-5\% der Betroffenen zuteil. Die Deutsche Gesellschaft für Pneumologie empfliehlt die pneumologische Rehabilitation als "unbedingt sinnvoll". 